\title{
A new strikingly coloured species of Siphonocryptus, sixth of its order (Diplopoda: Siphonocryptida)
}

\author{
HENRIK ENGHOFF \\ Natural History Museum of Denmark (Zoological Museum), University of Copenhagen, Universitetsparken 15, DK-2100 Kфbenhavn \\ Ø, Denmark.E-mail: henghoff@snm.ku.dk
}

The small millipede family Siphonocryptidae was revised by Enghoff \& Golovatch (1995) who assigned two genera and three species to it: Siphoncryptus compactus Pocock, 1894, from Sumatra, S. latior Enghoff \& Golovatch, 1995, from the Malaccan peninsula, and Hirudicryptus canariensis (Loksa, 1967) from the Canary and Madeiran archipelagos in the Atlantic Ocean. This highly disjunct distribution pattern has later become partly filled out by Hirudicryptus taiwanensis Korsós et al., 2008, from Taiwan, and H. quintumelementum Korsós et al., 2009, from Nepal.

Hoffman $(1977,1980)$ placed the Siphonocryptidae (at that time known only from Siphonocryptus compactus) in a separate suborder, Siphonocryptidea, in order Polyzoniida. This separation was supported by Enghoff \& Golovatch (1995). Shelley (2002) elevated this taxon to a full order, Siphonocryptida, based on an unpublished phylogenetic analysis by W.A. Shear. In the phylogenetic analysis by Sierwald \& Bond (2007), Siphonocryptida is sister to Platydesmida, and (Siphonocryptida + Platydesmida) is sister to Siphonophorida. Siphonocryptida is the second-smallest millipede order; only the Siphoniulida, with a mere 2 described species, is smaller (cf. Sierwald et al. 2003).

Siphonocryptids are rare in collections, and this is particularly true of the genus Siphonocryptus. Enghoff \& Golovatch (1995) knew of 4 specimens of $S$. compactus and 3 of $S$. latior. To my knowledge, no further specimens belonging to this genus have been reported, and the discovery of an eighth specimen, clearly differing from both described species, is therefore of considerable interest.

\section{Material and methods}

The single specimen of the new Siphonocryptus was found in a large material of small millipedes from the Cameron Highlands, continental Malaysia, collected by sifting by Petr Baňař (Moravian Museum, Brno) and kindly donated to the Natural History Museum of Denmark. This material is very rich in such millipede groups as glomeridesmids, glomerids, siphonorhinids, heterochordeumatids, metopidiotrichids, julids, paradoxosomatids and haplodesmids, and the fact that only one specimen of a siphonocryptid was found underlines the rarity of these millipedes. Photographs were taken with a BK Plus Lab Imaging System from Visionary Digital (http://www.visionarydigital.com) equipped with a Canon EOS 7D camera. Single images were combined with Helicon Focus (version 5.0) software from Helicon Soft Ltd., to increase depth of field.

\section{Siphonocryptus zigzag, new species}

(Figs. 1, 3)

Material studied. Holotype female, MALAYSIA, PAHANG, Cameron Highlands,"ORANGE ASLI vill.” env. Gunung Perdah [Mt.], $4^{\circ} 29.2 \mathrm{~N}, 101^{\circ} 22.1 \mathrm{E}, 1575 \mathrm{~m}$, sifting leaf litter in shallow ravine, 2-14.v.2009, Petr Baňař leg. (Natural History Museum of Denmark, Zoological Museum, University of Copenhagen, ZMUC 00101324).

Diagnosis. A species of Siphonocryptus with a dorsal colour pattern including two triangular lateral areas each covering 7 pleurotergites.

Description. Female holotype with 32 pleurotergites, body length $4.3 \mathrm{~mm}$, max. width $2.6 \mathrm{~mm}$. Dorsal colour pattern distinctive: ground colour pale yellowish, almost transparent along margins of body. Tergites 2-4 entirely dark brown. A mid-dorsal longitudinal band, running uninterrupted from tergite 5 to penultuimate tergum (both included) and 
covering ca. 1/6 of the body width, also dark brown. Lateral parts of tergites 14-31 medium brown. On most of these tergites the darkened areas extend to ca. halfway between lateral margin and middle of tergites, leaving a pale band ca. as broad as mid-dorsal dark band. On tergites 19-20, and again on tergites 26-27, however, the darkened areas become gradually narrower, resulting in a distinct-zig-zag boundary between dark and light (Fig. 1). On tergites 28-31, the darkened lateral areas extend almost to the median dark brown band, leaving only a narrow light parasagittal stripe. The last, large tergite 32 has narrow darkened lateral margins but is otherwise pale.

In all other respects similar to the other species in the genus.

Notes. Siphoncryptus zigzag differs from its two known congeners by its remarkable colour pattern. For comparison, the colour pattern of $S$. compactus is illustrated in Fig. 2. The colour pattern of $S$. zigzag will be further discussed in a forthcoming paper on comparable colour patterns in millipedes (Enghoff in prep.). In Fig. 3, the pleurotergite numbers and maximal body diameter of known specimens of Siphonocryptus are shown, based on the new species and data from Enghoff \& Golovatch (1995). Although the number of specimens is small, it looks as if S. zigzag is more similar to $S$. latior in body shape (very broad) than to $S$. compactus. The two former species have both been found in peninsular Malaysia, their type (and only known) localities lying ca. $125 \mathrm{~km}$ apart.
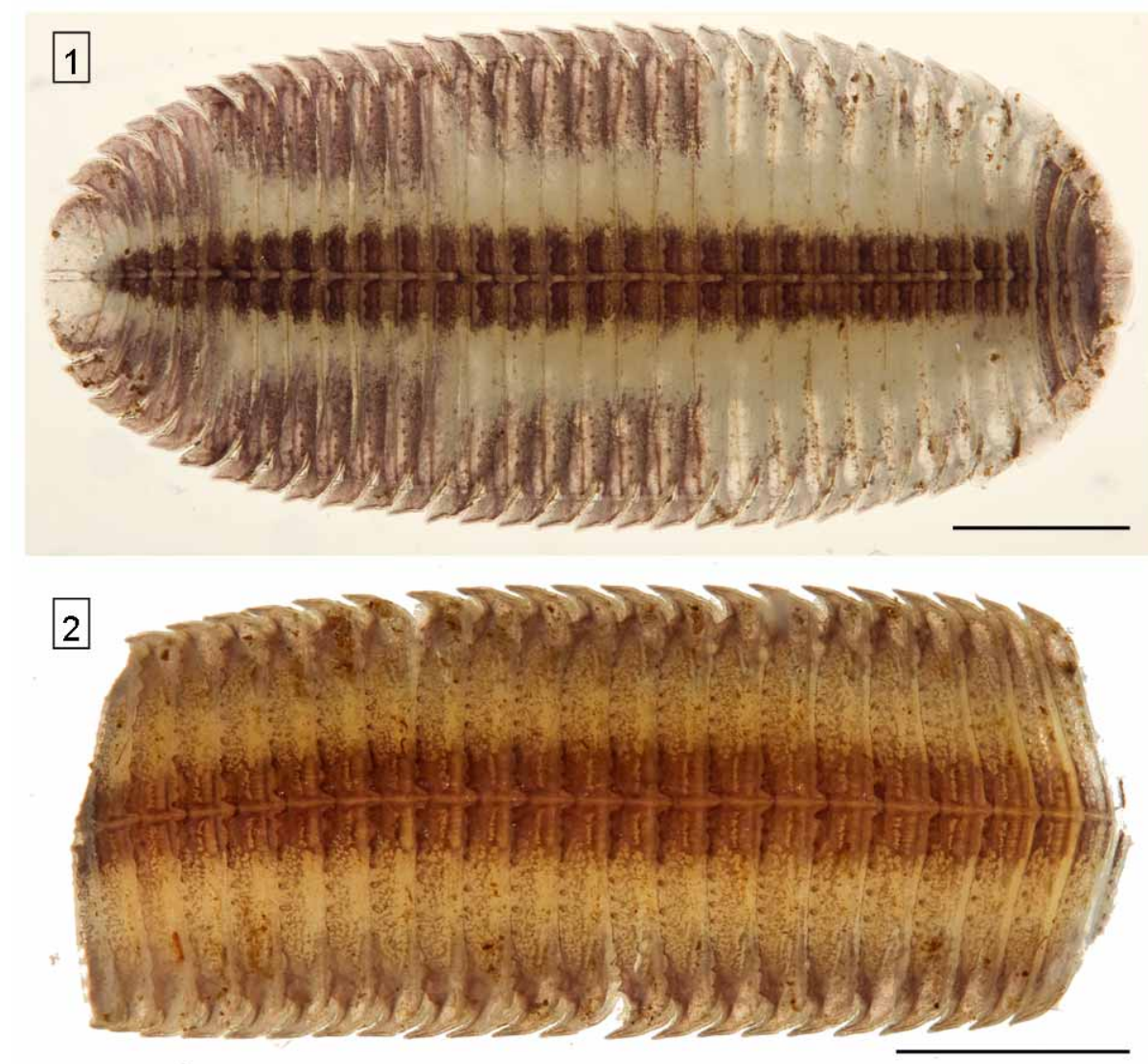

FIGURE 1-2. Siphonocryptus spp., dorsal view. 1: S zigzag n.sp., holotype; 2: S. compactus, specimen from MALAYSIA, W SUMATRA, Bukittinggi, Gn. Singgalang, 2100-2600 m, A. Riedel leg. (ZMUC 00101325), without front end, hind end not visible. Head end of both specimens to the right. Scale bars $=1 \mathrm{~mm}$.

\section{Acknowledgements}

I am grateful to Petr Ban̆ař (Moravian Museum, Brno) for the donation of his valuable millipede material, to Janet Beccaloni (Natural History Museum, London) for assistance with sorting the material, and to Nikolaj Scharff (Natural History Museum of Denmark) for photographic wizardry. 


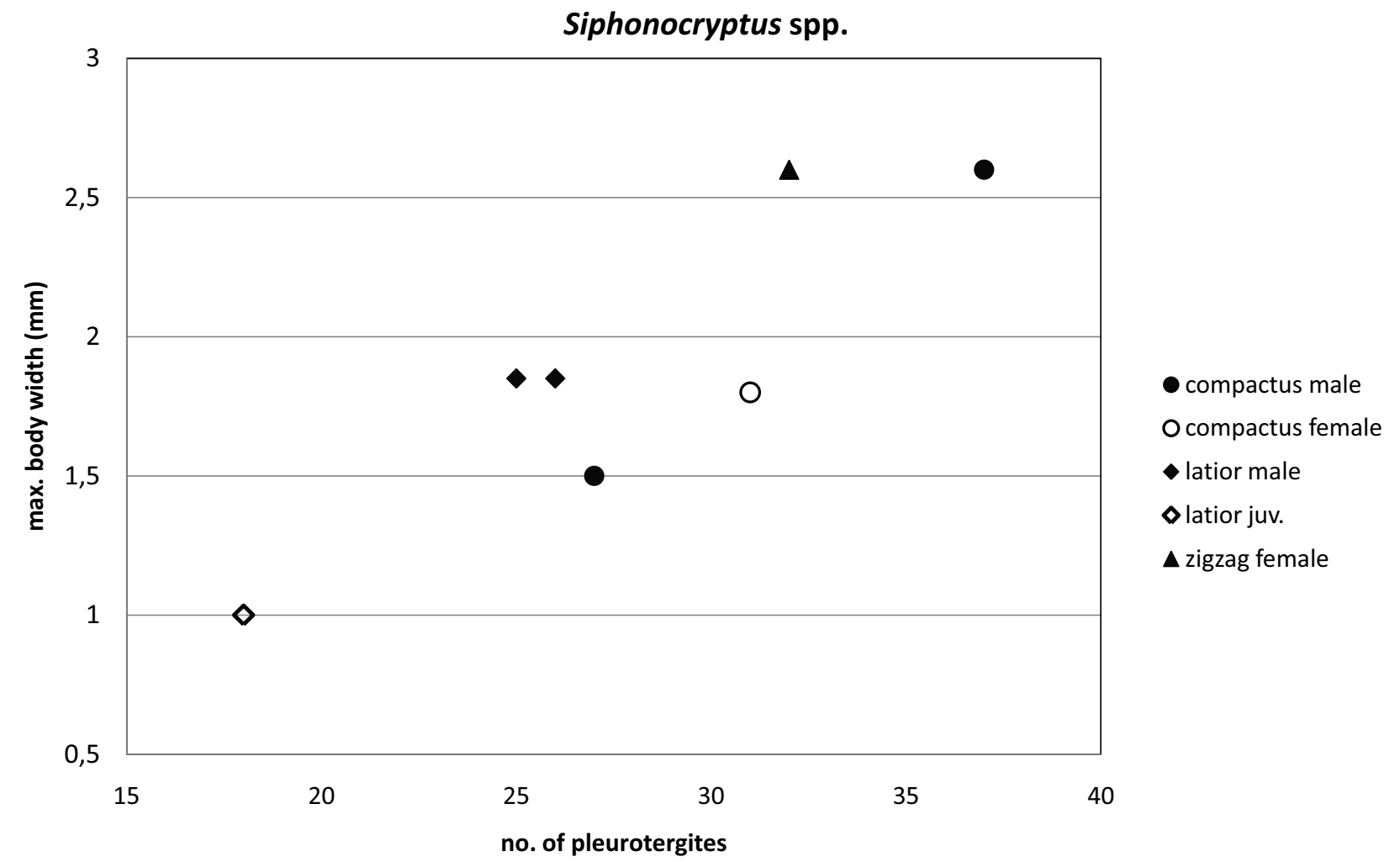

FIGURE 3. Relationship between number of pleurotergites and maximal body width in Siphonocryptus spp., based on holotype of S. zigzag and data from Enghoff \& Golovatch (1995).

\section{References}

Enghoff, H. \& Golovatch, S.I. (1995) A revision of the Siphonocryptidae (Diplopoda, Polyzoniida). Zoologica Scripta, 24, 29-41.

Hoffman, R.L. (1977) On the status of Siphonotus brasiliensis Brandt and of the diplopod family Siphonotidae (Polyzoniida). Deutsche entomologische Zeitschrift Neue Folge, 24, 425-431.

Hoffman, R.L. (1980) Classification of the Diplopoda. Muséum d'Histoire naturelle, Genève.

Korsós, Z., Enghoff, H. \& Chang, H.W. (2008) A most unusual animal distribution pattern: A new siphonocryptid millipede from Taiwan (Diplopoda, Siphonocryptida). Acta Zoologica Academiae Scientarum Hungaricae, 54, 151157.

Korsós, Z., Geoffroy, J.J. \& Mauriès, J.P. (2009) The fifth element: reconnection of the disjunct distribution of the members of Siphonocryptida (Diplopoda) with the description of a new species from Nepal. Journal of Natural History, 43, 435-445.

Shelley, R.M. (2002) A revised, annotated, family-level classification of the Diplopoda. Arthropoda Selecta, 11, 187207.

Sierwald, P. \& Bond, J.E. (2007) Current status of the myriapod class Diplopoda (Millipedes): Taxonomic diversity and phylogeny. Annual Review of Entomology, 52, 401-420.

Sierwald, P., Shear, W.A., Shelley, R-.M. \& Bond, J.E (2003) Millipede phylogeny revisited in the light of the enigmatic order Siphoniulida. Journal of zoological Systematics and Evolutionary Research, 41, 87-99. 